JOURNAL OF

FUNCTION SPACES AND APPLICATIONS

Volume 4, Number 2 (2006), 163-191
(C) 2006, Scientific Horizon http://www.jfsa.net

\title{
Three weights higher order Hardy type inequalities
}

\section{Aigerim A. Kalybay and Lars-Erik Persson}

(Communicated by Vladimir Stepanov)

2000 Mathematics Subject Classification. 26D10, 26D15.

Keywords and phrases. Inequalities, higher order Hardy type inequalities, kernels, weights, weighted differential operator.

Abstract. We investigate the following three weights higher order Hardy type inequality

$$
\|g\|_{q, u} \leq C\left\|D_{\rho}^{k} g\right\|_{p, v},
$$

where $D_{\rho}^{i}$ denotes the following weighted differential operator:

$$
D_{\rho}^{i} g(t)= \begin{cases}\frac{d^{i} g(t)}{d t^{i}}, & i=0,1, \ldots, m-1, \\ \frac{d^{i-m}}{d t^{i-m}}\left(\rho(t) \frac{d^{m} g(t)}{d t^{m}}\right), & i=m, m+1, \ldots, k,\end{cases}
$$

for a weight function $\rho(\cdot)$.

A complete description of the weights $u, v$ and $\rho$ so that (0.1) holds was given in [4] for the case $1<p \leq q<\infty$. Here the corresponding characterization is proved for the case $1<q<p<\infty$. The crucial step in the proof of the main result is to use a new Hardy type inequality (for a Volterra type operator), which we first state and prove. 


\section{Introduction}

Hardy type inequalities have been studied, developed and applied during the last decade in an almost unbelievable way. See, e.g., the monographs [7, 13 ] and the recent review article [6], completely devoted to this subject. In particular, some present knowledge about the higher order Hardy inequality is described in the book [7]. The weighted Hardy inequality for derivatives of order $k \geq 1$ has the following form:

$$
\|g\|_{q, u} \leq C\left\|g^{(k)}\right\|_{p, v} .
$$

Here $1<p, q<\infty$, the functions $u(\cdot)$ and $v(\cdot)$ are positive and locally integrable on $R_{+}=(0,+\infty)$ in the powers $q$ and $p$ respectively. Suppose also that $v^{-1}(\cdot) \in L_{p^{\prime}}^{\text {loc }}\left(R_{+}\right)$and $\|\cdot\|_{q, u}$ stands for:

$$
\|g\|_{q, u}=\left(\int_{0}^{\infty}|u(t) g(t)|^{q} d t\right)^{\frac{1}{q}}
$$

In this paper we shall consider a fairly new type of generalization of the Hardy type inequality (1.1), namely we investigate the estimate

$$
\|g\|_{q, u} \leq C\left\|D_{\rho}^{k} g\right\|_{p, v},
$$

where $D_{\rho}^{i}$ denotes the following weighted differential operator:

$$
D_{\rho}^{i} g(t)= \begin{cases}\frac{d^{i} g(t)}{d t^{i}}, & i=0,1, \ldots, m-1, \\ \frac{d^{i-m}}{d t^{i-m}}\left(\rho(t) \frac{d^{m} g(t)}{d t^{m}}\right), & i=m, m+1, \ldots, k,\end{cases}
$$

for a weight function $\rho(\cdot)$.

The introduced differential operator, where all derivatives are understood in the generalized sense, is called the $\rho$-weighted derivative of $g$ of the corresponding order $i=0,1, \ldots, k$.

This operator (even in more general form) was considered already in the twentieth by G. Polya [14] and, independently, in the thirtieth by G. Mammana [9]. See the important paper [8] by A.U. Levin and the references given there. This paper is also a source for us to obtain applications of our results. After this a number of authors have studied this operator; here we just mention works by R. Oinarov and B.L. Baideldinov (see e.g. [2, 3]), where it is, in particular, pointed out that this operator is interesting for some applications and even gives the basis for some new function spaces. 
We shall use the notations

$$
\begin{aligned}
& \lim _{t \rightarrow 0+} D_{\rho}^{i} g(t)=D_{\rho}^{i} g(0), \quad i=0,1, \ldots, k-1, \\
& \lim _{t \rightarrow \infty} D_{\rho}^{i} g(t)=D_{\rho}^{i} g(\infty), \quad i=0,1, \ldots, k-1,
\end{aligned}
$$

and consider the problem of finding necessary and sufficient conditions on the weights $u, v$, and $\rho$, under which the inequality (1.2) holds for all functions $g$ satisfying the "boundary conditions"

$$
\begin{aligned}
D_{\rho}^{i} g(0)=0, & i=0,1, \ldots, l-1, \\
D_{\rho}^{j} g(\infty) & =0, \quad j=l, l+1, \ldots, k-1,
\end{aligned}
$$

where $1 \leq l \leq k-1$.

In the paper [4] this problem was solved for the case $1<p \leq q<\infty$. In the same paper you can find several references and remarks concerning (1.1). We also refer to the fairly new $\mathrm{PhD}$ thesis of M. Nassyrova [10]. In particular, in [4] it was noted that in the paper [5] by A. Kufner and H.P. Heinig inequality (1.1) was studied under the setting of "boundary conditions" similar to those of the current consideration. Here we prove some corresponding characterizations for the case $1<q<p<\infty$. In particular, by putting $\rho=1$ in each of the characterizations we obtain the previous result of A. Kufner and H.P. Heinig [5].

The crucial step in the proofs of these main results is to use a Hardy type inequality

$$
\|K f\|_{q, u} \leq C\|f\|_{p, v}
$$

for a Volterra type operator of the following form:

$$
K f(t)=\int_{0}^{t} K(t, s) f(s) d s
$$

with the kernel

$$
K(t, s)=\int_{s}^{t}(t-x)^{\alpha-1} w(x) d x,
$$

where $\alpha>1$ is a real number.

Let $\mu$ be a non - negative Borel measure on $R_{+}$, then $\|\cdot\|_{q, \mu}$ now stands for

$$
\|g\|_{q, \mu}=\left(\int_{0}^{\infty}|g(x)|^{q} d \mu(x)\right)^{\frac{1}{q}} .
$$

Let us remark that in the case $d \mu(x)=u^{q}(x) d x$ the notation $\|\cdot\|_{q, \mu}$ coincides with the notation $\|\cdot\|_{q, u}$ introduced above. 
The paper is organized as follows: in Section 3 we present and prove our main result concerning the inequality (1.5) (see Theorem 1). In order not to disturb our discussions in the fairly technical proof of this theorem we have formulated and proved three useful lemmas in Section 2, where also some other preliminaries can be found. Finally, in Section 4 we use Theorem 1 to prove our main results connected to the inequality (1.2) (see Theorem 2, 3 and 4).

\section{Preliminaries}

Here and in the sequel $\frac{1}{p}+\frac{1}{p^{\prime}}=1, \frac{1}{q}+\frac{1}{q^{\prime}}=1(1<p, q<\infty)$. The symbol $X \ll Y$ means $X \leq c Y$ with some constant $c>0$, and the notation $X \approx Y$ asserts the existence of the two - sided estimate $X \ll Y \ll X$. Moreover, $Z$ stands for the set of all integers, and $Z_{+}$stands for the set of non - negative integers. Above and in the sequel $k, m, n, l \in Z_{+}$.

For $0 \leq t<b \leq \infty$ assume that $\mu([t, b)) \equiv \int_{t}^{b} d \mu(x)$.

Let $R$ denote an integral operator defined by

$$
R f(t)=\int_{0}^{t} r(t, s) f(s) d s
$$

with the kernel $r(t, s)=\int_{s}^{t} w(x) d x$.

It is easy to see that the kernels of the operators (1.6) and (2.1) are continuous, don't decrease in the first argument and don't increase in the second argument.

We now state and prove three useful technical lemmas.

Further for the operator (2.1) we shall need the following estimate:

$$
\|R f\|_{q, \mu} \ll A\|f\|_{p, v}, f \geq 0,
$$

where $\mu$ is some non - negative Borel measure. The kernel of (2.1) satisfies the condition $r(t, s)=r(t, x)+r(x, s)$, when $t \geq x \geq s \geq 0$, so in view of results in the paper [11] it follows the two - weighted estimate for (2.1) in the form (1.5). But in our case we need to prove the inequality in the form (2.2) because in the left side of (2.2) we have a Borel measure $\mu$.

Lemma 1. Let $1<q<p<\infty$. Then the estimate (2.2) holds with $A=\max \left\{A_{1}, A_{2}\right\}$, where

$$
A_{1}=\left(\int_{0}^{\infty}\left(\int_{t}^{\infty} d \mu(s)\right)^{\frac{p}{p-q}} d\left(\int_{0}^{t} r^{p^{\prime}}(t, s) v^{-p^{\prime}}(s) d s\right)^{\frac{q(p-1)}{p-q}}\right)^{\frac{p-q}{p q}}
$$




$$
A_{2}=\left(\int_{0}^{\infty}\left(\int_{t}^{\infty} r^{q}(s, t) d \mu(s)\right)^{\frac{p}{p-q}} d\left(\int_{0}^{t} v^{-p^{\prime}}(s) d s\right)^{\frac{q(p-1)}{p-q}}\right)^{\frac{p-q}{p q}} .
$$

Proof. Let $f \geq 0$. Then $R f$ is continuous, non-negative and nondecreasing on $R_{+}$, in addition, $(R f)(0)=0$. Hence, there exists a sequence of monotonously increasing points $\left\{t_{k}\right\}_{k \in Z_{0}}, Z_{0} \subset Z$, such that

$$
\begin{aligned}
(R f)\left(t_{k}\right) & =2^{k}, k \in Z_{0}, R_{+}=\bigcup_{k \in Z_{0}} I_{k}, I_{k}=\left[t_{k}, t_{k+1}\right), \\
I_{i} \bigcap I_{j} & =\varnothing \text { for } i \neq j, 2^{k} \leq(R f)(t) \leq 2^{k+1} \text { for } t \in I_{k},
\end{aligned}
$$

and $\forall k \in Z_{0}$

$$
2^{k-1}=2^{k}-2^{k-1}=\int_{t_{k-1}}^{t_{k}} r\left(t_{k}, s\right) f(s) d s+r\left(t_{k}, t_{k-1}\right) \int_{o}^{t_{k-1}} f(s) d s .
$$

If $N=\max \left\{k: k \in Z_{0}\right\}<\infty$, then suppose $t_{N+1}=\infty$.

The pointed out relations give:

$$
\begin{aligned}
\|R f\|_{q, \mu}^{q}= & \sum_{k \in Z_{0}} \int_{t_{k}}^{t_{k+1}}(R f)^{q}(t) d \mu(t) \\
\leq & 2^{2 q} \sum_{k \in Z_{0}} 2^{q(k-1)} \mu\left(\left[t_{k}, t_{k+1}\right)\right) \\
\ll & \sum_{k \in Z_{0}}\left(\int_{t_{k-1}}^{t_{k}} r\left(t_{k}, s\right) f(s) d s\right)^{q} \mu\left(\left[t_{k}, t_{k+1}\right)\right) \\
& +\sum_{k \in Z_{0}} r^{q}\left(t_{k}, t_{k-1}\right)\left(\int_{0}^{t_{k-1}} f(s) d s\right)^{q} \mu\left(\left[t_{k}, t_{k+1}\right)\right) \\
= & J_{1}^{0}+J_{2}^{0} .
\end{aligned}
$$

By twice using Hölder's inequality, we get

$$
\begin{aligned}
J_{1}^{0} \leq & \sum_{k \in Z_{0}}\left(\int_{t_{k-1}}^{t_{k}} r^{p^{\prime}}\left(t_{k}, s\right) v^{-p^{\prime}}(s) d s\right)^{\frac{q}{p^{\prime}}} \mu\left(\left[t_{k}, t_{k+1}\right)\right)\left(\int_{t_{k-1}}^{t_{k}}|v f|^{p} d s\right)^{\frac{q}{p}} \\
\leq & \left(\sum_{k \in Z_{0}}\left(\int_{t_{k-1}}^{t_{k}} r^{p^{\prime}}\left(t_{k}, s\right) v^{-p^{\prime}}(s) d s\right)^{\frac{p}{p-q}}\left(\mu\left(\left[t_{k}, t_{k+1}\right)\right)\right)^{\frac{p}{p-q}}\right)^{\frac{p-q}{p}} \\
& \times\left(\sum_{k \in Z_{0}} \int_{I_{k-1}}|v f|^{p} d s\right)^{\frac{q}{p}} .
\end{aligned}
$$


The functions $\int_{t_{k-1}}^{t} r^{p^{\prime}}(t, s) v^{-p^{\prime}}(s) d s$ and $\int_{0}^{t_{k-1}} r^{p^{\prime}}(t, s) v^{-p^{\prime}}(s) d s$ are non-decreasing and absolutely continuous on $I_{k-1}$, then

$$
\begin{aligned}
\left(\int_{t_{k-1}}^{t_{k}} r^{p^{\prime}}\left(t_{k}, s\right) v^{-p^{\prime}}(s) d s\right)^{\frac{q(p-1)}{p-q}} & =\int_{t_{k-1}}^{t_{k}} d\left(\int_{t_{k-1}}^{t} r^{p^{\prime}}(t, s) v^{-p^{\prime}}(s) d s\right)^{\frac{q(p-1)}{p-q}} \\
& \leq \int_{t_{k-1}}^{t_{k}} d\left(\int_{0}^{t} r^{p^{\prime}}(t, s) v^{-p^{\prime}}(s) d s\right)^{\frac{q(p-1)}{p-q}}
\end{aligned}
$$

Therefore, from (2.4) we have

$$
\begin{aligned}
J_{1}^{0} & \leq\left(\sum_{k \in Z_{0}} \int_{t_{k-1}}^{t_{k}}\left(\int_{t}^{\infty} d \mu(s)\right)^{\frac{p}{p-q}} d\left(\int_{0}^{t} r^{p^{\prime}}(t, s) v^{-p^{\prime}}(s) d s\right)^{\frac{q(p-1)}{p-q}}\right)^{\frac{p-q}{p}}\|f\|_{p, v}^{q} \\
& \leq A_{1}^{q}\|f\|_{p, v}^{q} .
\end{aligned}
$$

By introducing the measure $d \gamma_{0}(t)=\sum_{k \in Z_{0}} r^{q}\left(t_{k}, t_{k-1}\right) \mu\left(\left[t_{k}, t_{k+1}\right)\right)$ $\times \delta\left(t-t_{k-1}\right) d t$, where $\delta$ is the delta-function, the expression $J_{2}^{0}$ can be written in the following form:

$$
J_{2}^{0}=\int_{0}^{\infty}\left(\int_{0}^{t} f(s) d s\right)^{q} d \gamma_{0}(t)=\|H f\|_{q, \gamma_{0}}^{q} .
$$

Indeed,

$$
\begin{aligned}
\int_{0}^{\infty} & \left(\int_{0}^{t} f(s) d s\right)^{q} \sum_{k \in Z_{0}} r^{q}\left(t_{k}, t_{k-1}\right) \mu\left(\left[t_{k}, t_{k+1}\right)\right) \delta\left(t-t_{k-1}\right) d t \\
= & \sum_{k \in Z_{0}}\left(\int_{0}^{t_{k-1}} f(s) d s\right)^{q} r^{q}\left(t_{k}, t_{k-1}\right) \mu\left(\left[t_{k}, t_{k+1}\right)\right) \\
= & J_{2}^{0} .
\end{aligned}
$$

By using standard results from the theory of Hardy type inequalities (see e.g. [7]), we find that

$$
J_{2}^{0}=\|H f\|_{q, \gamma_{0}}^{q} \ll \widetilde{A}^{q}\|f\|_{p, v}^{q}
$$

where

$$
\widetilde{A}=\left(\int_{0}^{\infty}\left(\int_{t}^{\infty} d \gamma_{0}(s)\right)^{\frac{p}{p-q}} d\left(\int_{0}^{t} v^{-p^{\prime}}(s) d s\right)^{\frac{q(p-1)}{p-q}}\right)^{\frac{p-q}{p q}} .
$$


Now, we estimate separately the integral $\int_{t}^{\infty} d \gamma_{0}(s)$ in the following way:

$$
\begin{aligned}
\int_{t}^{\infty} d \gamma_{0}(s) & =\sum_{t_{k-1}>t} r^{q}\left(t_{k}, t_{k-1}\right) \mu\left(\left[t_{k}, t_{k+1}\right)\right) \\
& =\sum_{t_{k-1}>t} r^{q}\left(t_{k}, t_{k-1}\right) \int_{t_{k}}^{t_{k+1}} d \mu(s) \\
& \leq \sum_{t_{k-1}>t} \int_{t_{k}}^{t_{k+1}} r^{q}\left(s, t_{k-1}\right) d \mu(s) \\
& \leq \sum_{t_{k-1}>t} \int_{t_{k}}^{t_{k+1}} r^{q}(s, t) d \mu(s) \\
& \leq \int_{t}^{\infty} r^{q}(s, t) d \mu(s) .
\end{aligned}
$$

From this estimate we obtain $\widetilde{A} \leq A_{2}<\infty$. Consequently,

$$
J_{2}^{0} \ll A_{2}^{q}\|f\|_{p, v}^{q} .
$$

From (2.3), (2.5) and (2.6) we have that (2.2) holds.

To restrict the writing we suppose

$$
q(\alpha-1)\left(\int_{t}^{\infty}(x-t)^{q(\alpha-1)-1} u^{q}(x) d x\right) \equiv u_{1}^{q}(t) .
$$

Lemma 2. If $1<q, \alpha<\infty$, and $f \geq 0$, then

$$
\|R f\|_{q, u_{1}} \leq\|K f\|_{q, u} .
$$

Proof. By Fubini's theorem we have

$$
\begin{aligned}
\|R f\|_{q, u_{1}}^{q}= & \int_{0}^{\infty}\left(\int_{0}^{t}\left(\int_{s}^{t} w(y) d y\right) f(s) d s\right)^{q} \\
& \times d\left(-\int_{t}^{\infty}(x-t)^{q(\alpha-1)} u^{q}(x) d x\right) \\
= & q(\alpha-1) \int_{0}^{\infty}\left(\int_{0}^{t} w(y)\left(\int_{0}^{y} f(s) d s\right) d y\right)^{q} \\
& \times \int_{t}^{\infty}(x-t)^{q(\alpha-1)-1} u^{q}(x) d x d t \\
= & q(\alpha-1) \int_{0}^{\infty} \int_{0}^{x}(x-t)^{q(\alpha-1)-1}
\end{aligned}
$$




$$
\times\left(\int_{0}^{t} w(y)\left(\int_{0}^{y} f(s) d s\right) d y\right)^{q} d t u^{q}(x) d x .
$$

Applying Minkowski's inequality in (2.8) gives

$$
\begin{aligned}
\|R f\|_{q, u_{1}}^{q} \leq & q(\alpha-1) \int_{0}^{\infty}\left(\int_{0}^{x} w(y)\left(\int_{0}^{y} f(s) d s\right)\right. \\
& \left.\times\left(\int_{y}^{x}(x-t)^{q(\alpha-1)-1} d t\right)^{\frac{1}{q}} d y\right)^{q} u^{q}(x) d x \\
= & \int_{0}^{\infty}\left(\int_{0}^{x} w(y)\left(\int_{0}^{y} f(s) d s\right)(x-y)^{\alpha-1} d y\right)^{q} u^{q}(x) d x \\
= & \int_{0}^{\infty}\left(\int_{0}^{x} f(s)\left(\int_{s}^{x}(x-y)^{\alpha-1} w(y) d y\right) d s\right)^{q} u^{q}(x) d x \\
= & \|K f\|_{q, u}^{q} .
\end{aligned}
$$

Lemma 3. Suppose that the monotone function $f: R_{+} \rightarrow R_{+}$does not increase, the monotone function $\varphi: R_{+} \rightarrow R_{+}$does not decrease. Moreover, both of the functions are locally absolutely continuous on $R_{+}$and

$$
\lim _{t \rightarrow+\infty} f(t)=\lim _{t \rightarrow 0+} \varphi(t)=0 .
$$

Then the finiteness of any of the integrals

$$
\begin{gathered}
\int_{0}^{\infty} f(x) d \varphi(x), \\
\int_{0}^{\infty} \varphi(x) d(-f(x))
\end{gathered}
$$

implies the finiteness of the other one. Moreover,

$$
\int_{0}^{\infty} f(x) d \varphi(x)=\int_{0}^{\infty} \varphi(x) d(-f(x)) .
$$

Proof. Suppose that (2.9) is finite. Then, for any $t \in R_{+}$, we have

$$
\int_{0}^{t} f(x) d \varphi(x) \geq f(t) \int_{0}^{t} d \varphi(x)=f(t) \varphi(t),
$$

and, consequently, $\lim _{t \rightarrow 0+} f(t) \varphi(t)=0$.

Integrating by parts gives

$$
\int_{0}^{t} f(x) d \varphi(x)=f(t) \varphi(t)+\int_{0}^{t} \varphi(x) d(-f(x)), t \in R_{+} .
$$


By assumption the integral (2.9) is finite and, hence, the left side of (2.12) has a finite limit when $t \rightarrow+\infty$. We conclude that each summand on the right side of (2.12) has a finite limit when $t \rightarrow+\infty$ because they are nonnegative for all $t \in R_{+}$. Thus, the equality

$$
\int_{0}^{\infty} f(x) d \varphi(x)=\lim _{t \rightarrow+\infty} f(t) \varphi(t)+\int_{0}^{\infty} \varphi(x) d(-f(x))
$$

holds, i.e., the integral (2.10) is finite. Moreover, from the finiteness of (2.10) it follows that

$$
\int_{t}^{\infty} \varphi(x) d(-f(x)) \geq \varphi(t) \int_{t}^{\infty} d(-f(x))=\varphi(t) f(t) .
$$

Hence, $\lim _{t \rightarrow+\infty} \varphi(t) f(t)=0$, and this fact together with (2.13) yields (2.11).

Similarly, we can prove the finiteness of (2.9) from the finiteness of (2.10).

Remark 1. In all proofs in the sequel we more than once will use the operation of integration by parts, and for all these cases the conditions of Lemma 3 will hold. This implies that it is not necessary to every time refer to Lemma 3 and the equalities of (2.11) type can be written down automatically. Moreover, the conditions of the fulfillment of known inequalities will not be written down in the classical form but in the transformed form (cf. (2.11)), which is more suitable for our purposes.

\section{The main result for the inequality (1.5)}

Let

$$
\begin{aligned}
B_{1}= & \left(\int_{0}^{\infty}\left(\int_{0}^{t} K^{p^{\prime}}(t, s) v^{-p^{\prime}}(s) d s\right)^{\frac{q(p-1)}{p-q}}\left(\int_{t}^{\infty} u^{q}(x) d x\right)^{\frac{q}{p-q}} u^{q}(t) d t\right)^{\frac{p-q}{p q}}, \\
B_{2}= & \left(\int_{0}^{\infty}\left(\int_{t}^{\infty} K^{q}(x, t) u^{q}(x) d x\right)^{\frac{p}{p-q}} d\left(\int_{0}^{t} v^{-p^{\prime}}(s) d s\right)^{\frac{q(p-1)}{p-q}}\right)^{\frac{p-q}{p q}}, \\
B_{3}= & \left(\int_{0}^{\infty}\left(\int_{t}^{\infty}(x-t)^{q(\alpha-1)} u^{q}(x) d x\right)^{\frac{p}{p-q}}\right. \\
& \left.\times d\left(\int_{0}^{t}\left(\int_{s}^{t} w(x) d x\right)^{p^{\prime}} v^{-p^{\prime}}(s) d s\right)^{\frac{q(p-1)}{p-q}}\right)^{\frac{p-q}{p q}},
\end{aligned}
$$

and

$$
B=\max \left\{B_{1}, B_{2}, B_{3}\right\}
$$


Theorem 1. Let $1<q<p<\infty$. Then the estimate (1.5) holds if and only if $B<\infty$. In addition, $B \approx C$, where $C$ is the best constant in (1.5).

Proof. Sufficiency. Let $B<\infty$. It means that $B_{1}<\infty, B_{2}<\infty$ and $B_{3}<\infty$. Because of the non-negativeness of the kernel of the operator $K$ it is enough to prove the correctness of the inequality (1.5) for non-negative functions $(f \geq 0)$.

For $f \geq 0$ consider $F(x)=\int_{0}^{x} K(x, s) f(s) d s$. Then the function $F(x)$ is continuous and non-decreasing on $R_{+}$, and, moreover, $F(0)=0$. Assume that $h=\max \left\{1,2^{\alpha-2}\right\}$. Due to the properties of the function $F(x)$ on $R_{+}$ there exists a sequence of monotonously increasing points $\left\{x_{k}\right\}_{k \in Z_{1}} \subset R_{+}$, $Z_{1} \subset Z$, such that

$$
\begin{aligned}
x_{k} & =\sup \left\{x: F(x) \leq(h+1)^{k}\right\}, F\left(x_{k}\right)=(h+1)^{k}, I_{k}=\left[x_{k}, x_{k+1}\right), \\
R_{+} & =\bigcup_{k \in Z_{1}} I_{k},\left[x_{i}, x_{i+1}\right) \cap\left[x_{j}, x_{j+1}\right)=\varnothing \text { for } i \neq j .
\end{aligned}
$$

As before in Lemma 1 we suppose that if $N=\max \left\{k: k \in Z_{1}\right\}<\infty$, then $x_{N+1}=\infty$.

Consequently, $(h+1)^{k} \leq F(x) \leq(h+1)^{k+1}$ for $x \in I_{k}$, and

$$
\begin{aligned}
(h+1)^{k-1} & =(h+1)^{k}-h(h+1)^{k-1}=F\left(x_{k}\right)-h F\left(x_{k-1}\right) \\
& =\int_{0}^{x_{k}} K\left(x_{k}, s\right) f(s) d s-h \int_{0}^{x_{k-1}} K\left(x_{k-1}, s\right) f(s) d s \\
& =\int_{x_{k-1}}^{x_{k}} K\left(x_{k}, s\right) f(s) d s+\int_{0}^{x_{k-1}}\left[K\left(x_{k}, s\right)-h K\left(x_{k-1}, s\right)\right] f(s) d s .
\end{aligned}
$$

Moreover, since

$$
\begin{aligned}
K\left(x_{k}, s\right)-h K\left(x_{k-1}, s\right)= & \int_{s}^{x_{k}}\left(x_{k}-\tau\right)^{\alpha-1} w(\tau) d \tau \\
& -h \int_{s}^{x_{k-1}}\left(x_{k-1}-\tau\right)^{\alpha-1} w(\tau) d \tau \\
\leq & \int_{x_{k-1}}^{x_{k}}\left(x_{k}-\tau\right)^{\alpha-1} w(\tau) d \tau \\
& +h\left(x_{k}-x_{k-1}\right)^{\alpha-1} \int_{s}^{x_{k-1}} w(\tau) d \tau
\end{aligned}
$$

according to (3.1), we have

$$
(h+1)^{k-1} \leq \int_{x_{k-1}}^{x_{k}} K\left(x_{k}, s\right) f(s) d s+K\left(x_{k}, x_{k-1}\right)
$$




$$
\begin{aligned}
& \times \int_{0}^{x_{k-1}} f(s) d s+h\left(x_{k}-x_{k-1}\right)^{\alpha-1} \\
& \times \int_{0}^{x_{k-1}} r\left(x_{k-1}, s\right) f(s) d s .
\end{aligned}
$$

Taking into account (3.2), we can estimate $\|K f\|_{q, u}$ in the following way:

$$
\begin{aligned}
\|K f\|_{q, u}^{q}= & \sum_{k \in Z_{1}} \int_{I_{k}}|K f(x)|^{q} u^{q}(x) d x \\
= & \sum_{k \in Z_{1}} \int_{x_{k}}^{x_{k+1}} F^{q}(x) u^{q}(x) d x \\
\leq & \sum_{k \in Z_{1}}(h+1)^{q(k+1)} \int_{x_{k}}^{x_{k+1}} u^{q}(x) d x \\
= & (h+1)^{2 q} \sum_{k \in Z_{1}}(h+1)^{q(k-1)} \int_{x_{k}}^{x_{k+1}} u^{q}(x) d x \\
\ll & (h+1)^{2 q}\left(\sum_{k \in Z_{1}}\left(\int_{x_{k-1}}^{x_{k}} K\left(x_{k}, s\right) f(s) d s\right)^{q} \int_{x_{k}}^{x_{k+1}} u^{q}(s) d s\right. \\
& +\sum_{k \in Z_{1}}\left(K\left(x_{k}, x_{k-1}\right) \int_{0}^{x_{k-1}} f(s) d s\right)^{q} \int_{x_{k}}^{x_{k+1}} u^{q}(s) d s \\
& +h^{q} \sum_{k \in Z_{1}}\left(\left(x_{k}-x_{k-1}\right)^{\alpha-1} \int_{0}^{x_{k-1}} r\left(x_{k-1}, s\right) f(s) d s\right)^{q} \\
& \left.\times \int_{x_{k}}^{x_{k+1}} u^{q}(s) d s\right) .
\end{aligned}
$$

Denote by $J_{1}$ and $J_{2}$ the first two summands in the brackets of the last expression, respectively, and by $J_{3}$ the last summand without the constant $h^{q}$, and then estimate $J_{1}, J_{2}$, and $J_{3}$ separately.

We start with $J_{1}$. By using Hölder's inequality, we get

$$
\begin{aligned}
J_{1}= & \sum_{k \in Z_{1}}\left(\int_{x_{k-1}}^{x_{k}} K\left(x_{k}, s\right) f(s) d s\right)^{q} \int_{x_{k}}^{x_{k+1}} u^{q}(s) d s \\
\leq & \sum_{k \in Z_{1}}\left(\left(\int_{x_{k-1}}^{x_{k}} v^{p}(s) f^{p}(s) d s\right)^{\frac{1}{p}}\left(\int_{x_{k-1}}^{x_{k}} K^{p^{\prime}}\left(x_{k}, s\right) v^{-p^{\prime}}(s) d s\right)^{\frac{1}{p^{\prime}}}\right)^{q} \\
& \times \int_{x_{k}}^{x_{k+1}} u^{q}(s) d s
\end{aligned}
$$




$$
\begin{aligned}
\leq & \left(\sum_{k \in Z_{1}} \int_{x_{k-1}}^{x_{k}} v^{p}(s) f^{p}(s) d s\right)^{\frac{q}{p}} \\
& \left.\times\left(\sum_{k \in Z_{1}}\left(\int_{x_{k-1}}^{x_{k}} K^{p^{\prime}}\left(x_{k}, s\right) v^{-p^{\prime}}(s) d s\right)^{\frac{q(p-1)}{p-q}}\left(\int_{x_{k}}^{x_{k+1}} u^{q}(s) d s\right)\right)^{\frac{p}{p-q}}\right)^{\frac{p-q}{p}} .
\end{aligned}
$$

Furthermore,

$$
\begin{aligned}
\left(\int_{x_{k}}^{x_{k+1}} u^{q}(s) d s\right)^{\frac{p}{p-q}} & =\frac{p}{p-q} \int_{x_{k}}^{x_{k+1}}\left(\int_{t}^{x_{k+1}} u^{q}(s) d s\right)^{\frac{q}{p-q}} u^{q}(t) d t \\
& \left.\ll \int_{x_{k}}^{x_{k+1}}\left(\int_{t}^{\infty} u^{q}(s) d s\right)\right)^{\frac{q}{p-q}} u^{q}(t) d t
\end{aligned}
$$

and, from (3.3) we get

$$
\begin{aligned}
J_{1} & \leq\left(\int_{0}^{\infty}\left(\int_{0}^{t} K^{p^{\prime}}(t, s) v^{-p^{\prime}}(s) d s\right)^{\frac{q(p-1)}{p-q}}\left(\int_{t}^{\infty} u^{q}(s) d s\right)^{\frac{q}{p-q}} u^{q}(t) d t\right)^{\frac{p-q}{p}}\|f\|_{p, v}^{q} \\
& =B_{1}^{q}\|f\|_{p, v}^{q} .
\end{aligned}
$$

Hence,

$$
J_{1} \ll B_{1}^{q}\|f\|_{p, v}^{q} .
$$

Next, we note that, by assuming that

$$
d \gamma(t)=\sum_{k \in Z_{1}} K^{q}\left(x_{k}, x_{k-1}\right)\left(\int_{x_{k}}^{x_{k+1}} u^{q}(s) d s\right) \delta\left(t-x_{k-1}\right) d t
$$

where $\delta(\cdot)$ is the delta-function, $J_{2}$ can be written similarly as $J_{2}^{0}$ is written in Lemma 1, namely in the following form:

$$
J_{2}=\int_{0}^{\infty}\left(\int_{0}^{t} f(s) d s\right)^{q} d \gamma(t)=\|H f\|_{q, \gamma}^{q} .
$$

Therefore,

$$
J_{2}=\|H f\|_{q, \gamma}^{q} \ll \widetilde{B}_{1}^{q}\|f\|_{p, v}^{q}
$$

where

$$
\widetilde{B}_{1}=\left(\int_{0}^{\infty}\left(\int_{t}^{\infty} d \gamma(s)\right)^{\frac{p}{p-q}} d\left(\int_{0}^{t} v^{-p^{\prime}}(s) d s\right)^{\frac{q(p-1)}{p-q}}\right)^{\frac{p-q}{p q}}
$$


Now, we estimate separately the integral $\int_{t}^{\infty} d \gamma(s)$ in the following way:

$$
\begin{aligned}
\int_{t}^{\infty} d \gamma(s) & =\sum_{x_{k-1}>t} K^{q}\left(x_{k}, x_{k-1}\right) \int_{x_{k}}^{x_{k+1}} u^{q}(s) d s \\
& \leq \sum_{x_{k-1}>t} \int_{x_{k}}^{x_{k+1}} K^{q}\left(s, x_{k-1}\right) u^{q}(s) d s \\
& \leq \sum_{x_{k-1}>t} \int_{x_{k}}^{x_{k+1}} K^{q}(s, t) u^{q}(s) d s \\
& \leq \int_{t}^{\infty} K^{q}(s, t) u^{q}(s) d s .
\end{aligned}
$$

From this estimate we obtain $\widetilde{B}_{1} \leq B_{2}<\infty$. Consequently,

$$
J_{2} \ll B_{2}^{q}\|f\|_{p, v}^{q} .
$$

Now, we put

$$
d \lambda(t)=\sum_{k \in Z_{1}}\left(x_{k}-x_{k-1}\right)^{q(\alpha-1)} \int_{x_{k}}^{x_{k+1}} u^{q}(s) d s \delta\left(t-x_{k-1}\right) d t
$$

where $\delta(\cdot)$ is the delta-function. Then $J_{3}$ has the following form:

$$
J_{3}=\int_{0}^{\infty}\left(\int_{0}^{t} r(t, s) f(s) d s\right)^{q} d \lambda(t)=\|R f\|_{q, \lambda}^{q} .
$$

Indeed,

$$
\begin{aligned}
& \int_{0}^{\infty}\left(\int_{0}^{t} r(t, s) f(s) d s\right)^{q} \sum_{k \in Z_{1}}\left(x_{k}-x_{k-1}\right)^{q(\alpha-1)} \int_{x_{k}}^{x_{k+1}} u^{q}(s) d s \delta\left(t-x_{k-1}\right) d t \\
& \left.\quad=\sum_{k \in Z_{1}}\left(\int_{0}^{x_{k-1}} r\left(x_{k-1}, s\right) f(s) d s\right)^{q}\left(x_{k}-x_{k-1}\right)^{q(\alpha-1)} \int_{x_{k}}^{x_{k+1}} u^{q}(s) d s\right) \\
& =J_{3} .
\end{aligned}
$$

Due to Lemma 1 we have

$$
J_{3}=\|R f\|_{q, \lambda}^{q} \ll \max \left\{\widetilde{A}_{1}^{q}, \widetilde{A}_{2}^{q}\right\}\|f\|_{p, v}^{q}
$$

where

$$
\widetilde{A}_{1}=\left(\int_{0}^{\infty}\left(\int_{t}^{\infty} d \lambda(s)\right)^{\frac{p}{p-q}} d\left(\int_{0}^{t} r^{p^{\prime}}(t, s) v^{-p^{\prime}}(s) d s\right)^{\frac{q(p-1)}{p-q}}\right)^{\frac{p-q}{p q}} .
$$




$$
\widetilde{A}_{2}=\left(\int_{0}^{\infty}\left(\int_{t}^{\infty} r^{q}(s, t) d \lambda(s)\right)^{\frac{p}{p-q}} d\left(\int_{0}^{t} v^{-p^{\prime}}(s) d s\right)^{\frac{q(p-1)}{p-q}}\right)^{\frac{p-q}{p q}}
$$

We estimate separately the following integral in the expression $\widetilde{A}_{2}$ :

$$
\begin{aligned}
\int_{t}^{\infty} r^{q}(s, t) d \lambda(s) & =\sum_{x_{k-1}>t}\left(x_{k}-x_{k-1}\right)^{q(\alpha-1)} r^{q}\left(x_{k-1}, t\right) \int_{x_{k}}^{x_{k+1}} u^{q}(s) d s \\
& =\sum_{x_{k-1}>t}\left(\int_{t}^{x_{k-1}} w(\tau)\left(x_{k}-x_{k-1}\right)^{\alpha-1} d \tau\right)^{q} \int_{x_{k}}^{x_{k+1}} u^{q}(s) d s \\
& \leq \sum_{x_{k-1}>t}\left(\int_{t}^{x_{k}} w(\tau)\left(x_{k}-\tau\right)^{\alpha-1} d \tau\right)^{q} \int_{x_{k}}^{x_{k+1}} u^{q}(s) d s \\
& \leq \sum_{x_{k-1}>t} \int_{x_{k}}^{x_{k+1}}\left(\int_{t}^{s} w(\tau)(s-\tau)^{\alpha-1} d \tau\right)^{q} u^{q}(s) d s \\
& \leq \int_{t}^{\infty} r^{q}(s, t) u^{q}(s) d s .
\end{aligned}
$$

This estimate gives $\widetilde{A}_{2} \leq B_{2}<\infty$.

Moreover, since

$$
\begin{aligned}
\int_{t}^{\infty} d \lambda(s) & =\sum_{x_{k-1}>t}\left(x_{k}-x_{k-1}\right)^{q(\alpha-1)} \int_{x_{k}}^{x_{k+1}} u^{q}(s) d s \\
& \leq \int_{t}^{\infty}(s-t)^{q(\alpha-1)} u^{q}(s) d s,
\end{aligned}
$$

we have $\widetilde{A}_{1} \leq B_{3}<\infty$.

We put the obtained estimates for $\widetilde{A}_{2}$ and $\widetilde{A}_{1}$ into (3.6) and get

$$
J_{3} \ll \max \left\{B_{2}^{q}, B_{3}^{q}\right\}\|f\|_{p, v}^{q}
$$

By combining (3.4), (3.5), and (3.7) we see that (1.5) holds, and, in addition, $C \ll B$, where $C$ is the best constant in (1.5).

Necessity. Suppose that the inequality (1.5) holds with the best constant $C>0$. Then it is well - known that the dual inequality

$$
\left\|v^{-1} K^{\prime} g\right\|_{p^{\prime}} \leq C\left\|u^{-1} g\right\|_{q^{\prime}}
$$

holds, where

$$
K^{\prime} g(s)=\int_{s}^{\infty} K(t, s) g(t) d t
$$

(see e.g. [7]). 
Take the following test function

$$
g_{1}(t)=\chi_{(a, b)}\left(\int_{t}^{b} u^{q}(s) d s\right)^{\frac{q-1}{p-q}}\left(\int_{a}^{t} K^{p^{\prime}}(t, s) v^{-p^{\prime}}(s) d s\right)^{\frac{(p-1)(q-1)}{p-q}} u^{q}(t)
$$

for any $a, b: 0<a<b<\infty$, and put it into the right side of the inequality (3.8) and we obtain that

$$
\begin{aligned}
\left\|u^{-1} g_{1}\right\|_{q^{\prime}}^{q^{\prime}}= & \int_{0}^{\infty}\left(\chi_{(a, b)}\left(\int_{t}^{b} u^{q}(x) d x\right)^{\frac{q-1}{p-q}}\right. \\
& \left.\times\left(\int_{a}^{t} K^{p^{\prime}}(t, s) v^{-p^{\prime}}(s) d s\right)^{\frac{(p-1)(q-1)}{p-q}} u^{q}(t)\right)^{q^{\prime}} u^{-q^{\prime}}(t) d t \\
= & \int_{a}^{b}\left(\int_{t}^{b} u^{q}(x) d x\right)^{\frac{q}{p-q}}\left(\int_{a}^{t} K^{p^{\prime}}(t, s) v^{-p^{\prime}}(s) d s\right)^{\frac{q(p-1)}{p-q}} u^{q}(t) d t .
\end{aligned}
$$

Next we insert $g_{1}(\cdot)$ into the left side of (3.8) and find that

$$
\begin{aligned}
\left\|v^{-1} K^{\prime} g_{1}\right\|_{p^{\prime}}^{p^{\prime}} & \geq \int_{a}^{b} v^{-p^{\prime}}(t)\left(\int_{t}^{b} K(s, t) g_{1}(s) d s\right)^{p^{\prime}} d t \\
& =p^{\prime} \int_{a}^{b} v^{-p^{\prime}}(t) \int_{t}^{b} K(s, t) g_{1}(s)\left(\int_{s}^{b} K(x, t) g_{1}(x) d x\right)^{p^{\prime}-1} d s d t .
\end{aligned}
$$

Hence, by Fubini's theorem,

$$
\left\|v^{-1} K^{\prime} g_{1}\right\|_{p^{\prime}}^{p^{\prime}} \geq p^{\prime} \int_{a}^{b} g_{1}(s) \int_{a}^{s} v^{-p^{\prime}}(t) K(s, t)\left(\int_{s}^{b} K(x, t) g_{1}(x) d x\right)^{p^{\prime}-1} d t d s .
$$

If $x \geq s$, then $K(x, t) \geq K(s, t)$. Using this fact and (3.10) we get

$$
\left\|v^{-1} K^{\prime} g_{1}\right\|_{p^{\prime}}^{p^{\prime}} \geq p^{\prime} \int_{a}^{b} g_{1}(s) \int_{a}^{s} v^{-p^{\prime}}(t) K^{p^{\prime}}(s, t)\left(\int_{s}^{b} g_{1}(x) d x\right)^{p^{\prime}-1} d t d s .
$$

Next we estimate the integral $\int_{s}^{b} g_{1}(x) d x$ in the brackets of (3.11):

$$
\begin{aligned}
& \int_{s}^{b} g_{1}(x) d x \\
& \quad=\int_{s}^{b}\left(\int_{x}^{b} u^{q}(\tau) d \tau\right)^{\frac{q-1}{p-q}}\left(\int_{a}^{x} K^{p^{\prime}}(x, \tau) v^{-p^{\prime}}(\tau) d \tau\right)^{\frac{(p-1)(q-1)}{p-q}} u^{q}(x) d x
\end{aligned}
$$




$$
\begin{aligned}
& \geq\left(\int_{a}^{s} K^{p^{\prime}}(s, \tau) v^{-p^{\prime}}(\tau) d \tau\right)^{\frac{(p-1)(q-1)}{p-q}}\left(\int_{s}^{b}\left(\int_{x}^{b} u^{q}(\tau) d \tau\right)^{\frac{q-1}{p-q}} u^{q}(x) d x\right) \\
& =\left(\int_{a}^{s} K^{p^{\prime}}(s, \tau) v^{-p^{\prime}}(\tau) d \tau\right)^{\frac{(p-1)(q-1)}{p-q}}\left(\frac{p-q}{p-1}\right)\left(\int_{s}^{b} u^{q}(t) d t\right)^{\frac{p-1}{p-q}} .
\end{aligned}
$$

By using this estimate in (3.11) we find that

$$
\begin{aligned}
\left\|v^{-1} K^{\prime} g_{1}\right\|_{p^{\prime}}^{p^{\prime}} \geq & p^{\prime}\left(\frac{p-q}{p-1}\right)^{\left(p^{\prime}-1\right)} \int_{a}^{b}\left(\int_{s}^{b} u^{q}(t) d t\right)^{\frac{q-1}{p-q}} \\
& \times\left(\int_{a}^{s} K^{p^{\prime}}(s, t) v^{-p^{\prime}}(t) d t\right)^{\frac{(p-1)(q-1)}{p-q}} \int_{a}^{s} K^{p^{\prime}}(s, t) v^{-p^{\prime}}(t) d t \\
& \times\left(\int_{a}^{s} K^{p^{\prime}}(s, t) v^{-p^{\prime}}(t) d t\right)^{\frac{(p-1)(q-1)\left(p^{\prime}-1\right)}{p-q}} \\
& \times\left(\int_{s}^{b} u^{q}(t) d t\right)^{\frac{(p-1)\left(p^{\prime}-1\right)}{p-q}} u^{q}(s) d s,
\end{aligned}
$$

so that

$$
\begin{aligned}
\left\|v^{-1} K^{\prime} g_{1}\right\|_{p^{\prime}}^{p^{\prime}} \geq & p^{\prime}\left(\frac{p-q}{p-1}\right)^{\left(p^{\prime}-1\right)} \int_{a}^{b}\left(\int_{s}^{b} u^{q}(t) d t\right)^{\frac{q}{p-q}} \\
& \times\left(\int_{a}^{s} K^{p^{\prime}}(s, t) v^{-p^{\prime}}(t) d t\right)^{\frac{q(p-1)}{p-q}} u^{q}(s) d s .
\end{aligned}
$$

From (3.8), (3.9), and (3.12) it follows

$$
\left(\int_{a}^{b}\left(\int_{s}^{b} u^{q}(t) d t\right)^{\frac{q}{p-q}}\left(\int_{a}^{s} K^{p^{\prime}}(s, t) v^{-p^{\prime}}(t) d t\right)^{\frac{q(p-1)}{p-q}} u^{q}(s) d s\right)^{\frac{p-q}{p q}} \ll C .
$$

If in the last estimated expression we proceed to limits when $a \rightarrow 0$ and $b \rightarrow \infty$, then, by also taking into account $(2.11)$, we get $B_{1} \ll C$.

Next, we consider the test function

$$
f_{1}(t)=\chi_{(a, b)}(t)\left(\int_{t}^{b} K^{q}(s, t) u^{q}(s) d s\right)^{\frac{1}{p-q}}\left(\int_{a}^{t} v^{-p^{\prime}}(\tau) d \tau\right)^{\frac{q-1}{p-q}} v^{-p^{\prime}}(t)
$$

for any $a, b: 0<a<b<\infty$.

Put $f_{1}(\cdot)$ into the right side of the inequality (1.5):

$$
\left\|f_{1}\right\|_{p, v}^{p}=\int_{0}^{\infty}\left(v(t) \chi_{(a, b)}(t)\left(\int_{t}^{b} K^{q}(s, t) u^{q}(s) d s\right)^{\frac{1}{p-q}}\right.
$$




$$
\begin{aligned}
& \left.\times\left(\int_{a}^{t} v^{-p^{\prime}}(\tau) d \tau\right)^{\frac{q-1}{p-q}} v^{-p^{\prime}}(t)\right)^{p} d t \\
= & \int_{a}^{b}\left(\int_{t}^{b} K^{q}(s, t) u^{q}(s) d s\right)^{\frac{p}{p-q}}\left(\int_{a}^{t} v^{-p^{\prime}}(\tau) d \tau\right)^{\frac{p(q-1)}{p-q}} v^{-p^{\prime}}(t) d t .
\end{aligned}
$$

Moreover, we insert $f_{1}(\cdot)$ into the left side of (1.5). Arguing as before for the test function $g_{1}(\cdot)$, we get

$$
\begin{aligned}
\left\|K f_{1}\right\|_{q, u}^{q}= & \int_{0}^{\infty}\left(\int_{0}^{t} K(t, s) f_{1}(s) d s\right)^{q} u^{q}(t) d t \\
= & q \int_{0}^{\infty} \int_{0}^{t}\left(\int_{0}^{x} K(t, s) f_{1}(s) d s\right)^{q-1} K(t, x) f_{1}(x) d x u^{q}(t) d t \\
\geq & q \int_{a}^{b} \int_{a}^{t}\left(\int_{a}^{x} K(t, s) f_{1}(s) d s\right)^{q-1} K(t, x) f_{1}(x) d x u^{q}(t) d t \\
= & q \int_{a}^{b} f_{1}(x) \int_{x}^{b}\left(\int_{a}^{x} K(t, s) f_{1}(s) d s\right)^{q-1} K(t, x) u^{q}(t) d t d x \\
\geq & q \int_{a}^{b} f_{1}(x) \int_{x}^{b} K^{q}(t, x) u^{q}(t) d t\left(\int_{a}^{x} f_{1}(s) d s\right)^{q-1} d x \\
\geq & q \int_{a}^{b} f_{1}(x) \int_{x}^{b} K^{q}(t, x) u^{q}(t) d t\left(\int_{a}^{x}\left(\int_{s}^{b} K^{q}(\tau, s) u^{q}(\tau) d \tau\right)^{\frac{1}{p-q}}\right. \\
& \left.\times\left(\int_{a}^{s} v^{-p^{\prime}}(\tau) d \tau\right)^{\frac{q-1}{p-q}} v^{-p^{\prime}}(s) d s\right)^{q-1} d x \\
\geq & q \int_{a}^{b} f_{1}(x) \int_{x}^{b} K^{q}(t, x) u^{q}(t) d t\left(\int_{x}^{b} K^{q}(\tau, x) u^{q}(\tau) d \tau\right)^{\frac{q-1}{p-q}} \\
& \times\left(\int_{a}^{x}\left(\int_{a}^{s} v^{-p^{\prime}}(\tau) d \tau\right)^{\frac{q-1}{p-q}} v^{-p^{\prime}}(s) d s\right)^{q-1} d x \\
= & q\left(\frac{p-q}{p-1}\right)^{q-1} \int_{a}^{b} f_{1}(x)\left(\int_{x}^{b} K^{q}(\tau, x) u^{q}(\tau) d \tau\right)^{\frac{p-1}{p-q}} \\
& \times\left(\int_{a}^{x} v^{-p^{\prime}}(\tau) d \tau\right) \frac{(p-1)(q-1)}{p-q} d x \\
&
\end{aligned}
$$




$$
\begin{aligned}
= & q\left(\frac{p-q}{p-1}\right)^{q-1} \int_{a}^{b}\left(\int_{x}^{b} K^{q}(\tau, x) u^{q}(\tau) d \tau\right)^{\frac{p}{p-q}} \\
& \times\left(\int_{a}^{x} v^{-p^{\prime}}(\tau) d \tau\right)^{\frac{p(q-1)}{p-q}} v^{-p^{\prime}}(x) d x .
\end{aligned}
$$

According to (1.5), (3.13), and (3.14) we have

$$
\left(\int_{a}^{b}\left(\int_{t}^{b} K^{q}(s, t) u^{q}(s) d s\right)^{\frac{p}{p-q}}\left(\int_{a}^{t} v^{-p^{\prime}}(\tau) d \tau\right)^{\frac{p(q-1)}{p-q}} v^{-p^{\prime}}(t) d t\right)^{\frac{p-q}{p q}} \ll C .
$$

If in the last estimated expression we proceed to limits when $a \rightarrow 0$ and $b \rightarrow \infty$, then, by taking into account (2.11), we get $B_{2} \ll C$.

From (1.5) and (2.7) we get

$$
\|R f\|_{q, u_{1}} \leq C\|f\|_{p, v} .
$$

As before, we note that the dual inequality

$$
\left\|v^{-1} R^{\prime} g\right\|_{p^{\prime}} \leq C\left\|u_{1}^{-1} g\right\|_{q^{\prime}}
$$

holds, where

$$
R^{\prime} g(s)=\int_{s}^{\infty} r(t, s) g(t) d t
$$

Next, we put the test function

$$
g_{2}(t)=\chi_{(a, b)}\left(\int_{t}^{b} u_{1}^{q}(s) d s\right)^{\frac{q-1}{p-q}}\left(\int_{a}^{t} r^{p^{\prime}}(t, s) v^{-p^{\prime}}(s) d s\right)^{\frac{(p-1)(q-1)}{p-q}} u_{1}^{q}(t)
$$

into the right and left sides of the inequality (3.15), where $a, b: 0<a<$ $b<\infty$. Then, by arguing in the same way as in the previous cases, we obtain

$$
\left\|u_{1}^{-1} g_{2}\right\|_{q^{\prime}}^{q^{\prime}}=\int_{a}^{b}\left(\int_{t}^{b} u_{1}^{q}(s) d s\right)^{\frac{q}{p-q}}\left(\int_{a}^{t} r^{p^{\prime}}(t, s) v^{-p^{\prime}}(s) d s\right)^{\frac{q(p-1)}{p-q}} u_{1}^{q}(t) d t,
$$

$$
\begin{aligned}
\left\|v^{-1} R^{\prime} g_{2}\right\|_{p^{\prime}}^{p^{\prime}} & \gg \int_{a}^{b} g_{2}(t) \int_{a}^{t} r^{p^{\prime}}(t, s) v^{-p^{\prime}}(s) d s\left(\int_{t}^{b} g_{2}(s) d s\right)^{p^{\prime}-1} d t \\
& \gg \int_{a}^{b}\left(\int_{t}^{b} u_{1}^{q}(s) d s\right)^{\frac{q}{p-q}}\left(\int_{a}^{t} r^{p^{\prime}}(t, s) v^{-p^{\prime}}(s) d s\right)^{\frac{q(p-1)}{p-q}} u_{1}^{q}(t) d t .
\end{aligned}
$$


From (1.5), (3.16), and (3.17) we get

$$
\left(\int_{a}^{b}\left(\int_{t}^{b} u_{1}^{q}(s) d s\right)^{\frac{q}{p-q}}\left(\int_{a}^{t} r^{p^{\prime}}(t, s) v^{-p^{\prime}}(s) d s\right)^{\frac{q(p-1)}{p-q}} u_{1}^{q}(t) d t\right)^{\frac{p-q}{p q}} \ll C .
$$

If in the last estimated expression we proceed to limits when $a \rightarrow 0$ and $b \rightarrow \infty$, then, by taking into account (2.11) and

$$
\int_{t}^{\infty} u_{1}^{q}(s) d s=\int_{t}^{\infty}(x-t)^{q(\alpha-1)} u^{q}(x) d x
$$

we get $B_{3} \ll C$.

Thus we have showed that $B_{1} \ll C, B_{2} \ll C$, and $B_{3} \ll C$, and, hence, $B \ll C$. This estimate together with the estimate $C \ll B$ we proved in the sufficient part give $B \approx C$.

Remark 2. The kernel $K(t, s)=\int_{s}^{t}(t-x)^{\alpha-1} w(x) d x$ for integer $\alpha$ can be written down in the form

$$
K(t, s)=(\alpha-1) ! \int_{s}^{t} w\left(x_{\alpha}\right) \int_{x_{\alpha}}^{t} \int_{x_{\alpha-1}}^{t} \ldots \int_{x_{2}}^{t} d x_{1} d x_{2} \ldots d x_{\alpha} .
$$

In the paper [1] sufficient conditions and in the paper [12] necessary conditions of the validity of the inequality (1.5) were found in the case when the kernel of the integral operator has the form (3.18). In fact, it was proved that the correctness of (1.5) depends on $\alpha$ conditions. However, as we have proved in a direct way in this paper we need only three conditions.

Remark 3. Lemma 2 and Theorem 1 can be formulated also for the conjugate integral operators. In fact, the proofs are similar so we omit both the formulations and the proofs.

\section{The main results for the inequality (1.2)}

We divide the studying of the problem into three cases: (1) $m<l$; (2) $m>l$; (3) $m=l$.

Theorem 2. Let $1<q<p<\infty, k \geq 3$ and $1 \leq m<l<k$. Suppose that the function $g$ satisfies the conditions (1.3) and (1.4). Then the inequality (1.2) holds with a finite constant $C>0$ independent of $g$ if and only if the following conditions hold:

$$
\left(\int_{0}^{\infty}\left(\int_{0}^{t} v^{-p^{\prime}}(s)\left(\int_{0}^{t} \rho^{-1}(x) \varphi(s, x) d x\right)^{p^{\prime}} d s\right)^{\frac{q(p-1)}{p-q}}\right.
$$




$$
\left.\times\left(\int_{t}^{\infty} u^{q}(s)(s-t)^{q(m-1)} d s\right)^{\frac{q}{p-q}} d\left(-\int_{t}^{\infty} u^{q}(s)(s-t)^{q(m-1)} d s\right)\right)^{\frac{p-q}{p q}}<\infty,
$$

$$
\begin{aligned}
& \left(\int_{0}^{\infty}\left(\int_{0}^{t} v^{-p^{\prime}}(s)\left(\int_{0}^{t} \rho^{-1}(x) \varphi(s, x)(t-x)^{m-1} d x\right)^{p^{\prime}} d s\right)^{\frac{q(p-1)}{p-q}}\right. \\
& \left.\quad \times\left(\int_{t}^{\infty} u^{q}(s) d s\right)^{\frac{q}{p-q}} u^{q}(t) d t\right)^{\frac{p-q}{p q}}<\infty,
\end{aligned}
$$

where $\varphi(s, x)= \begin{cases}x^{l-m} s^{k-l-1}, & x \leq s, \\ x^{l-m-1} s^{k-l}, & x>s,\end{cases}$

$$
\begin{aligned}
& \left(\int_{0}^{\infty}\left(\int_{t}^{\infty} u^{q}(s)\left(\int_{t}^{s} \rho^{-1}(x) x^{l-m-1}(s-x)^{m-1} d x\right)^{q} d s\right)^{\frac{p}{p-q}}\right. \\
& \left.\quad \times\left(\int_{0}^{t} v^{-p^{\prime}}(s) s^{p^{\prime}(k-l)} d s\right)^{\frac{p(q-1)}{p-q}} v^{-p^{\prime}}(t) t^{p^{\prime}(k-l)} d t\right)^{\frac{p-q}{p q}}<\infty
\end{aligned}
$$

and

$$
\begin{aligned}
\text { (4.4) }\left(\int_{0}^{\infty}\right. & \left(\int_{0}^{t} u^{q}(s)\left(\int_{0}^{s} \rho^{-1}(x) x^{l-m}(s-x)^{m-1} d x\right)^{q} d s\right)^{\frac{p}{p-q}} \\
& \left.\times\left(\int_{t}^{\infty} v^{-p^{\prime}}(s) s^{p^{\prime}(k-l-1)} d s\right)^{\frac{p(q-1)}{p-q}} v^{-p^{\prime}}(t) t^{p^{\prime}(k-l-1)} d t\right)^{\frac{p-q}{p q}}<\infty .
\end{aligned}
$$

Proof. If we assume $F(t)=D_{\rho}^{k} g(t)$, then, according to conditions (1.3) and (1.4), we have

$$
g(t)=B \int_{0}^{t} \int_{\tau}^{\infty} F(s)(s-\tau)^{k-l-1} \int_{\tau}^{t} \rho^{-1}(x)(x-\tau)^{l-m-1}(t-x)^{m-1} d x d s d \tau,
$$

where $B=(-1)^{k-l} /(k-l-1) !(l-m-1) !(m-1)$ !.

In the paper [4] it was proved that

$$
\begin{aligned}
g(t) \approx & \int_{0}^{t} F(s) \int_{0}^{s} \rho^{-1}(x)(t-s)^{m-1} s^{k-l-1} x^{l-m} d x d s \\
& +\int_{0}^{t} F(s) \int_{0}^{s} \rho^{-1}(x)(s-x)^{m-1} s^{k-l-1} x^{l-m} d x d s \\
& +\int_{0}^{t} F(s) \int_{s}^{t} \rho^{-1}(x)(t-x)^{m-1} s^{k-l} x^{l-m-1} d x d s \\
& +\int_{t}^{\infty} F(s) \int_{0}^{t} \rho^{-1}(x)(t-x)^{m-1} s^{k-l-1} x^{l-m} d x d s .
\end{aligned}
$$


If we use the notations $\mathbf{K}_{i} F(t), i=1,2,3,4$, for the each summand of the above expression, then the fulfillment of the inequality (1.2) is equal to the simultaneous fulfillment of four inequalities:

$$
\left\|\mathbf{K}_{i} F\right\|_{q, u} \leq C_{i}\|F\|_{p, v}, i=1,2,3,4 .
$$

We study each of these estimates (4.5) separately.

We start with the inequality (4.5) for the operator $\mathbf{K}_{1}$. If we put $\Phi(s)=s^{k-l-1} F(s) \int_{0}^{s} \rho^{-1}(x) x^{l-m} d x$, then we can rewrite (4.5) in the following form:

$$
\begin{aligned}
& \left(\int_{0}^{\infty}\left(u(t) \int_{0}^{t}(t-s)^{m-1} \Phi(s) d s\right)^{q} d t\right)^{\frac{1}{q}} \\
& \quad \leq C_{1}\left(\int_{0}^{\infty}\left(\Phi(s)\left[v(s) s^{l-k+1}\left(\int_{0}^{s} \rho^{-1}(x) x^{l-m} d x\right)^{-1}\right]\right)^{p} d s\right)^{\frac{1}{p}}
\end{aligned}
$$

Since the operator $\mathbf{K}_{1}$ with the kernel $K_{1}(t, s)=(t-s)^{m-1}$ is an operator of Riemann-Liouville type, then, in view of results in the paper [15], the inequality (4.6) holds if and only if

$$
\begin{aligned}
& \left(\int_{0}^{\infty}\left(\int_{t}^{\infty} u^{q}(s)(s-t)^{q(m-1)} d s\right)^{\frac{p}{p-q}}\right. \\
& \left.\quad \times d\left(\int_{0}^{t} v^{-p^{\prime}}(s) s^{p^{\prime}(k-l-1)}\left(\int_{0}^{s} \rho^{-1}(x) x^{l-m} d x\right)^{p^{\prime}} d s\right)^{\frac{q(p-1)}{p-q}}\right)^{\frac{p-q}{p q}} \\
& \approx\left(\int_{0}^{\infty}\left(\int_{0}^{t} v^{-p^{\prime}}(s) s^{p^{\prime}(k-l-1)}\left(\int_{0}^{s} \rho^{-1}(x) x^{l-m} d x\right)^{p^{\prime}} d s\right)^{\frac{q(p-1)}{p-q}}\right. \\
& \left.\quad \times\left(\int_{t}^{\infty} u^{q}(s)(s-t)^{q(m-1)} d s\right)^{\frac{q}{p-q}} d\left(-\int_{t}^{\infty} u^{q}(s)(s-t)^{q(m-1)} d s\right)\right)^{\frac{p-q}{p q}} \\
& <\infty
\end{aligned}
$$

and

$$
\begin{gathered}
\left(\int_{0}^{\infty}\left(\int_{0}^{t} v^{-p^{\prime}}(s) s^{p^{\prime}(k-l-1)}(t-s)^{p^{\prime}(m-1)}\left(\int_{0}^{s} \rho^{-1}(x) x^{l-m} d x\right)^{p^{\prime}} d s\right)^{\frac{q(p-1)}{p-q}}\right. \\
\left.\times\left(\int_{t}^{\infty} u^{q}(s) d s\right)^{\frac{q}{p-q}} u^{q}(t) d t\right)^{\frac{p-q}{p q}}<\infty .
\end{gathered}
$$


Now, consider (4.5) for $i=2$. Put $\Psi(s)=s^{k-l-1} F(s) \int_{0}^{s} \rho^{-1}(x)$ $\times(s-x)^{m-1} x^{l-m} d x$. Then the considered estimate takes the form:

$$
\begin{aligned}
& \left(\int_{0}^{\infty}\left(u(t) \int_{0}^{t} \Psi(s) d s\right)^{q} d t\right)^{\frac{1}{q}} \\
& \quad \leq C_{2}\left(\int_{0}^{\infty}\left(\Psi(s)\left[v(s) s^{l-k+1}\left(\int_{0}^{s} \rho^{-1}(x)(s-x)^{m-1} x^{l-m} d x\right)^{-1}\right]\right)^{p} d s\right)^{\frac{1}{p}} .
\end{aligned}
$$

Of course, the inequality (4.9) is a standard Hardy type inequality, which holds if and only if (see e.g. [7]):

$$
\begin{gathered}
\left(\int_{0}^{\infty}\left(\int_{0}^{t} v^{-p^{\prime}}(s) s^{p^{\prime}(k-l-1)}\left(\int_{0}^{s} \rho^{-1}(x) x^{l-m}(s-x)^{m-1} d x\right)^{p^{\prime}} d s\right)^{\frac{q(p-1)}{p-q}}\right. \\
\left.\times\left(\int_{t}^{\infty} u^{q}(s) d s\right)^{\frac{q}{p-q}} u^{q}(t) d t\right)^{\frac{p-q}{p q}}<\infty
\end{gathered}
$$

Consider (4.5) for the operator $\mathbf{K}_{3}$. It is obvious that the kernel $K_{3}(t, s)=\int_{s}^{t} \rho^{-1}(x)(t-x)^{m-1} x^{l-m-1} d x$ has the form of the kernel from Theorem 1. Therefore, the inequality (4.5) for $i=3$ holds if and only if the condition (4.3) and two conditions

$$
\begin{gathered}
\left(\int_{0}^{\infty}\left(\int_{0}^{t} v^{-p^{\prime}}(s) s^{p^{\prime}(k-l)}\left(\int_{s}^{t} \rho^{-1}(x) x^{l-m-1}(t-x)^{m-1} d x\right)^{p^{\prime}} d s\right)^{\frac{q(p-1)}{p-q}}\right. \\
\left.\times\left(\int_{t}^{\infty} u^{q}(s) d s\right)^{\frac{q}{p-q}} u^{q}(t) d t\right)^{\frac{p-q}{p q}}<\infty
\end{gathered}
$$

and

$$
\begin{aligned}
& \left(\int_{0}^{\infty}\left(\int_{t}^{\infty} u^{q}(s)(s-t)^{q(m-1)} d s\right)^{\frac{p}{p-q}}\right. \\
& \left.\quad \times d\left(\int_{0}^{t} v^{-p^{\prime}}(s) s^{p^{\prime}(k-l)}\left(\int_{s}^{t} \rho^{-1}(x) x^{l-m-1} d x\right)^{p^{\prime}} d s\right)^{\frac{q(p-1)}{p-q}}\right)^{\frac{p-q}{p q}} \\
& \approx\left(\int_{0}^{\infty}\left(\int_{0}^{t} v^{-p^{\prime}}(s) s^{p^{\prime}(k-l)}\left(\int_{s}^{t} \rho^{-1}(x) x^{l-m-1} d x\right)^{p^{\prime}} d s\right)^{\frac{q(p-1)}{p-q}}\right.
\end{aligned}
$$




$$
\left.\times\left(\int_{t}^{\infty} u^{q}(s)(s-t)^{q(m-1)} d s\right)^{\frac{q}{p-q}} d\left(-\int_{t}^{\infty} u^{q}(s)(s-t)^{q(m-1)} d s\right)\right)^{\frac{p-q}{p q}}<\infty
$$

hold.

It is easy to show that the combination of the conditions (4.7) and (4.12) in fact is equivalent to (4.1), and that the combination of (4.8), (4.10), and (4.11) gives (4.2). Moreover, the inequality (4.5) for $i=4$ is a standard Hardy type inequality and for its correctness the condition (4.4) is necessary and sufficient (see e.g. [7]).

Theorem 3. Let $1<q<p<\infty, k \geq 3$ and $1 \leq l<m<k$. Suppose that the function $g$ satisfies the conditions (1.3) and (1.4). Then the inequality (1.2) holds with a finite constant $C>0$ independent of $g$ if and only if the following conditions hold:

$$
\begin{aligned}
& \left(\int_{0}^{\infty}\left(\int_{0}^{t} u^{q}(s)\left(\int_{0}^{t} \rho^{-1}(x)(t-x)^{n-1} \psi(s, x) d x\right)^{q} d s\right)^{\frac{p}{p-q}}\right. \\
& \left.\times\left(\int_{t}^{\infty} v^{-p^{\prime}}(s) d s\right)^{\frac{p(q-1)}{p-q}} v^{-p^{\prime}}(t) d t\right)^{\frac{p-q}{p q}}<\infty \\
& \left(\int_{0}^{\infty}\left(\int_{0}^{t} u^{q}(s)\left(\int_{0}^{t} \rho^{-1}(x) \psi(s, x) d x\right)^{q} d s\right)^{\frac{p}{p-q}}\right. \\
& \times\left(\int_{t}^{\infty} v^{-p^{\prime}}(s)(s-t)^{p^{\prime}(n-1)} d s\right)^{\frac{p(q-1)}{p-q}} \\
& \left.\times d\left(-\int_{t}^{\infty} v^{-p^{\prime}}(s)(s-t)^{p^{\prime}(n-1)} d s\right)\right)^{\frac{p-q}{p q}}<\infty
\end{aligned}
$$

where $\psi(s, x)= \begin{cases}x^{m-l} s^{l-1}, & x \leq s, \\ x^{m-l-1} s^{l}, & x>s\end{cases}$

$$
\begin{gathered}
\left(\int_{0}^{\infty}\left(\int_{t}^{\infty} v^{-p^{\prime}}(s)\left(\int_{t}^{s} \rho^{-1}(x)(s-x)^{n-1} x^{m-l-1} d x\right)^{p^{\prime}} d s\right)^{\frac{q(p-1)}{p-q}}\right. \\
\left.\times\left(\int_{0}^{t} u^{q}(s) s^{q l} d s\right)^{\frac{q}{p-q}} u^{q}(t) t^{q l} d t\right)^{\frac{p-q}{p q}}<\infty
\end{gathered}
$$

and

$$
\begin{aligned}
& \left(\int _ { 0 } ^ { \infty } ( \int _ { t } ^ { \infty } u ^ { q } ( s ) s ^ { q ( l - 1 ) } d s ) ^ { \frac { p } { p - q } } d \left(\int_{0}^{t} v^{-p^{\prime}}(s)\right.\right. \\
& \left.\left.\quad \times\left(\int_{0}^{s} \rho^{-1}(x) x^{m-l}(s-x)^{n-1} d x\right)^{p^{\prime}} d s\right)^{\frac{q(p-1)}{p-q}}\right)^{\frac{p-q}{p q}}<\infty
\end{aligned}
$$


Proof. Let $F(t)=D_{\rho}^{k} g(t)$. Then, due to the conditions (1.3) and (1.4), we have

$g(t)=B \int_{0}^{t}(t-s)^{l-1} \int_{s}^{\infty} \rho^{-1}(x)(x-s)^{m-l-1} \int_{x}^{\infty}(\tau-x)^{n-1} F(\tau) d \tau d x d s$,

where $B=(-1)^{k-l} /(n-1) !(m-l-1) !(l-1)$ !.

In the paper [4] it was proved that

$$
\begin{aligned}
g(t) \approx & t^{l-1} \int_{0}^{t} F(\tau) \int_{0}^{\tau} \rho^{-1}(x)(\tau-x)^{n-1} x^{m-l} d x d \tau \\
& +t^{l-1} \int_{t}^{\infty} F(\tau) \int_{0}^{t} \rho^{-1}(x)(\tau-t)^{n-1} x^{m-l} d x d \tau \\
& +t^{l-1} \int_{t}^{\infty} F(\tau) \int_{0}^{t} \rho^{-1}(x)(t-x)^{n-1} x^{m-l} d x d \tau \\
& +t^{l} \int_{t}^{\infty} F(\tau) \int_{t}^{\tau} \rho^{-1}(x)(\tau-x)^{n-1} x^{m-l-1} d x d \tau .
\end{aligned}
$$

Insert this expression instead of $g$ in the inequality (1.2) and we get four (4.5) type inequalities, the simultaneous fulfillment of which is equivalent to the validity of (1.2).

Since the first inequality is a standard Hardy type inequality, the condition (4.16) is necessary and sufficient for it correctness (see e.g. [7]).

The integral operator of the second inequality with the kernel $K_{2}(\tau, t)=$ $(\tau-t)^{n-1}$ is an operator of Riemann-Liouville type, and, in view of the results in the paper [15], it holds if and only if

$$
\begin{gathered}
\left(\int_{0}^{\infty}\left(\int_{0}^{t} u^{q}(s) s^{q(l-1)}(t-s)^{q(n-1)}\left(\int_{0}^{s} \rho^{-1}(x) x^{m-l} d x\right)^{q} d s\right)^{\frac{p}{p-q}}\right. \\
\left.\times\left(\int_{t}^{\infty} v^{-p^{\prime}}(s) d s\right)^{\frac{p(q-1)}{p-q}} v^{-p^{\prime}}(t) d t\right)^{\frac{p-q}{p q}}<\infty
\end{gathered}
$$

and

$$
\begin{aligned}
& \left(\int_{0}^{\infty}\left(\int_{t}^{\infty} v^{-p^{\prime}}(s)(s-t)^{p^{\prime}(n-1)} d s\right)^{\frac{q(p-1)}{p-q}}\right. \\
& \left.\quad \times d\left(\int_{0}^{t} u^{q}(s) s^{q(l-1)}\left(\int_{0}^{s} \rho^{-1}(x) x^{m-l} d x\right)^{q} d s\right)^{\frac{p}{p-q}}\right)^{\frac{p-q}{p q}}
\end{aligned}
$$




$$
\begin{aligned}
\approx & \left(\int_{0}^{\infty}\left(\int_{0}^{t} u^{q}(s) s^{q(l-1)}\left(\int_{0}^{s} \rho^{-1}(x) x^{m-l} d x\right)^{q} d s\right)^{\frac{p}{p-q}}\right. \\
& \times\left(\int_{t}^{\infty} v^{-p^{\prime}}(s)(s-t)^{p^{\prime}(n-1)} d s\right)^{\frac{p(q-1)}{p-q}} \\
& \left.\times d\left(-\int_{t}^{\infty} v^{-p^{\prime}}(s)(s-t)^{p^{\prime}(n-1)} d s\right)\right)^{\frac{p-q}{p q}}<\infty
\end{aligned}
$$

The third inequality is again a standard Hardy type inequality, which is correct if and only if (see e.g. [7]):

$$
\begin{gathered}
\left(\int_{0}^{\infty}\left(\int_{0}^{t} u^{q}(s) s^{q(l-1)}\left(\int_{0}^{s} \rho^{-1}(x)(s-x)^{n-1} x^{m-l} d x\right)^{q} d s\right)^{\frac{p}{p-q}}\right. \\
\left.\times\left(\int_{t}^{\infty} v^{-p^{\prime}}(s) d s\right)^{\frac{p(q-1)}{p-q}} v^{-p^{\prime}}(t) d t\right)^{\frac{p-q}{p q}}<\infty .
\end{gathered}
$$

The kernel $K_{4}(\tau, t)=\int_{t}^{\tau} \rho^{-1}(x)(\tau-x)^{n-1} x^{m-l-1} d x$ of the integral operator of the fourth inequality has the form of the kernel from Theorem 1 , but the operator itself is conjugate to the operator from Theorem 1 . Therefore, by taking into account Remark 3 we can assert that it is valid if and only if condition (4.15) and the following two conditions

$$
\begin{gathered}
\left(\int_{0}^{\infty}\left(\int_{0}^{t} u^{q}(s) s^{q l}\left(\int_{s}^{t} \rho^{-1}(x)(t-x)^{n-1} x^{m-l-1} d x\right)^{q} d s\right)^{\frac{p}{p-q}}\right. \\
\left.\times\left(\int_{t}^{\infty} v^{-p^{\prime}}(s) d s\right)^{\frac{p(q-1)}{p-q}} v^{-p^{\prime}}(t) d t\right)^{\frac{p-q}{p q}}<\infty
\end{gathered}
$$

and

$$
\begin{aligned}
& \left(\int_{0}^{\infty}\left(\int_{t}^{\infty} v^{-p^{\prime}}(s)(s-t)^{p^{\prime}(n-1)} d s\right)^{\frac{q(p-1)}{p-q}}\right. \\
& \left.\quad \times d\left(\int_{0}^{t} u^{q}(s) s^{q l}\left(\int_{s}^{t} \rho^{-1}(x) x^{m-l-1} d x\right)^{q} d s\right)^{\frac{p}{p-q}}\right)^{\frac{p-q}{p q}} \\
& \approx\left(\int_{0}^{\infty}\left(\int_{0}^{t} u^{q}(s) s^{q l}\left(\int_{s}^{t} \rho^{-1}(x) x^{m-l-1} d x\right)^{q} d s\right)^{\frac{p}{p-q}}\right. \\
& \quad \times\left(\int_{t}^{\infty} v^{-p^{\prime}}(s)(s-t)^{p^{\prime}(n-1)} d s\right)^{\frac{p(q-1)}{p-q}} \\
& \left.\quad \times d\left(-\int_{t}^{\infty} v^{-p^{\prime}}(s)(s-t)^{p^{\prime}(n-1)} d s\right)\right)^{\frac{p-q}{p q}}<\infty
\end{aligned}
$$


hold.

It is obvious that the combination of the conditions (4.17), (4.19) and (4.20) is equivalent to (4.13), and that the combination of (4.18) and (4.21) gives (4.14).

Theorem 4. Let $1<q<p<\infty, k \geq 2$ and $1 \leq m=l<k$. Suppose that the function $g$ satisfies the conditions (1.3) and (1.4). Then the inequality (1.2) holds with a finite constant $C>0$ independent of $g$ if and only if the following conditions hold:

$$
\begin{aligned}
& \left(\int_{0}^{\infty}\left(\int_{t}^{\infty} u^{q}(s)(s-t)^{q(m-1)} d s\right)^{\frac{p}{p-q}}\right. \\
& \left.\times d\left(\int_{0}^{t} v^{-p^{\prime}}(s)\left(\int_{0}^{s} \rho^{-1}(\tau)(s-\tau)^{n-1} d \tau\right)^{p^{\prime}} d s\right)^{\frac{q(p-1)}{p-q}}\right)^{\frac{p-q}{p q}}<\infty
\end{aligned}
$$

$$
\left(\int_{0}^{\infty}\left(\int_{0}^{t} v^{-p^{\prime}}(s)\left(\int_{0}^{s} \rho^{-1}(\tau)(t-\tau)^{m-1}(s-\tau)^{n-1} d \tau\right)^{p^{\prime}} d s\right)^{\frac{q(p-1)}{p-q}}\right.
$$

$$
\begin{aligned}
& \left.\times\left(\int_{t}^{\infty} u^{q}(s) d s\right)^{\frac{q}{p-q}} u^{q}(t) d t\right)^{\frac{p-q}{p q}}<\infty \\
& \left(\int_{0}^{\infty}\left(\int_{0}^{t} u^{q}(s)\left(\int_{0}^{s} \rho^{-1}(\tau)(t-\tau)^{n-1}(s-\tau)^{m-1} d \tau\right)^{q} d s\right)^{\frac{p}{p-q}}\right. \\
& \left.\times\left(\int_{t}^{\infty} v^{-p^{\prime}}(s) d s\right)^{\frac{p(q-1)}{p-q}} v^{-p^{\prime}}(t) d t\right)^{\frac{p-q}{p q}}<\infty
\end{aligned}
$$

and

$$
\begin{aligned}
& \left(\int_{0}^{\infty}\left(\int_{t}^{\infty} v^{-p^{\prime}}(s)(s-t)^{p^{\prime}(n-1)} d s\right)^{\frac{q(p-1)}{p-q}}\right. \\
& \left.\times d\left(\int_{0}^{t} u^{q}(s)\left(\int_{0}^{s} \rho^{-1}(\tau)(s-\tau)^{m-1} d \tau\right)^{q} d s\right)^{\frac{p}{p-q}}\right)^{\frac{p-q}{p q}}<\infty .
\end{aligned}
$$

Proof. We put $F(t)=D_{\rho}^{k} g(t)$. According to the conditions (1.3) and (1.4) we have

$$
g(t)=B \int_{0}^{t}(t-\tau)^{m-1} \rho^{-1}(\tau) \int_{\tau}^{\infty}(s-\tau)^{n-1} F(s) d s d \tau
$$

where $B=(-1)^{n} /(n-1) !(m-1) !$.

From [4] it is known that

$$
g(t) \approx \int_{0}^{t} F(s)(t-s)^{m-1} \int_{0}^{s} \rho^{-1}(\tau)(s-\tau)^{n-1} d \tau d s
$$




$$
\begin{aligned}
& +\int_{0}^{t} F(s) \int_{0}^{s} \rho^{-1}(\tau)(s-\tau)^{k-2} d \tau d s \\
& +\int_{t}^{\infty} F(s)(s-t)^{n-1} \int_{0}^{t} \rho^{-1}(\tau)(t-\tau)^{m-1} d \tau d s \\
& +\int_{t}^{\infty} F(s) \int_{0}^{t} \rho^{-1}(\tau)(t-\tau)^{k-2} d \tau d s .
\end{aligned}
$$

Inserting this expression into (1.2) leads to four (4.5) type inequalities.

Since the integral operators of the first and third inequalities are operators of Riemann-Liouville type, then the results of the paper [15] allow us to write down necessary and sufficient conditions of their validity (condition (4.22) with the first condition written down below relates to the first inequality, and condition (4.25) with the second below condition relates to the third inequality):

$$
\begin{gathered}
\left(\int_{0}^{\infty}\left(\int_{0}^{t} v^{-p^{\prime}}(s)(t-s)^{p^{\prime}(m-1)}\left(\int_{0}^{s} \rho^{-1}(\tau)(s-\tau)^{n-1} d \tau\right)^{p^{\prime}} d s\right)^{\frac{q(p-1)}{p-q}}\right. \\
\left.\times\left(\int_{t}^{\infty} u^{q}(s) d s\right)^{\frac{q}{p-q}} u^{q}(t) d t\right)^{\frac{p-q}{p q}}<\infty
\end{gathered}
$$

and

$$
\begin{gathered}
\left(\int_{0}^{\infty}\left(\int_{0}^{t} u^{q}(s)(t-s)^{q(n-1)}\left(\int_{0}^{s} \rho^{-1}(\tau)(s-\tau)^{m-1} d \tau\right)^{q} d s\right)^{\frac{p}{p-q}}\right. \\
\left.\times\left(\int_{t}^{\infty} v^{-p^{\prime}}(s) d s\right)^{\frac{p(q-1)}{p-q}} v^{-p^{\prime}}(t) d t\right)^{\frac{p-q}{p q}}<\infty
\end{gathered}
$$

The second and fourth summands lead to standard Hardy type inequalities, which hold respectively if and only if (see e.g. [7]):

$$
\begin{aligned}
& \left(\int_{0}^{\infty}\left(\int_{0}^{t} v^{-p^{\prime}}(s)\left(\int_{0}^{s} \rho^{-1}(\tau)(s-\tau)^{k-2} d \tau\right)^{p^{\prime}} d s\right)^{\frac{q(p-1)}{p-q}}\right. \\
& \left.\quad \times\left(\int_{t}^{\infty} u^{q}(s) d s\right)^{\frac{q}{p-q}} u^{q}(t) d t\right)^{\frac{p-q}{p q}}<\infty \\
& \left(\int_{0}^{\infty}\left(\int_{0}^{t} u^{q}(s)\left(\int_{0}^{s} \rho^{-1}(\tau)(s-\tau)^{k-2} d \tau\right)^{q} d s\right)^{\frac{p}{p-q}}\right. \\
& \left.\quad \times\left(\int_{t}^{\infty} v^{-p^{\prime}}(s) d s\right)^{\frac{p(q-1)}{p-q}} v^{-p^{\prime}}(t) d t\right)^{\frac{p-q}{p q}}<\infty .
\end{aligned}
$$

The combination of the conditions (4.26) and (4.28) is equivalent to the condition (4.23) and the combination of (4.27) and (4.29) gives (4.24). 
Acknowledgements. We thank the careful referee and Professor Vladimir Stepanov for some generous advices, which have improved the final version of this paper. In particular, the short proof of Lemma 2 is due to Stepanov.

\section{References}

[1] A. O. Baiarystanov, Two-weighted estimate of multiple integration operator with weights, Vestnik Kaz. State Univ., ser.: mat., mech., and inf., 12 (1998), 13-20 (Russian).

[2] B. L. Baideldinov, On going to generalized polynomial of function with integrable weighted derivative, Dokl. Rus. Acad. Sci., 354 (3) (1997), 295-297 (Russian).

[3] B. L. Baideldinov and R. Oinarov, Properties of one weighted space, Proc. Conf. "Function Spaces, Theory of Approximations and Nonlinear Analysis", Moscow, 1995, 49 (Russian).

[4] A.A. Kalybay, A generalization of the weighted Hardy inequality for one class of integral operators, Siberian Math. J., 45 (1) (2004), 100-112.

[5] A. Kufner and H. P. Heinig, Hardy inequality for higher order derivatives, Trudy Mat. Inst. Steklov, 192 (1990), 105-113 (Russian); English transl.: Proc. Steklov Inst. Math., 192 (1992), 113-121.

[6] A. Kufner, L. Maligranda and L.-E. Persson, The prehistory of the Hardy inequality, Amer. Math. Monthly, to appear (17 pages).

[7] A. Kufner and L.-E. Persson, Weighted Inequalities of Hardy Type, World Scientific, New Jersey/London/Singapore/Hong Kong, 2003.

[8] A. U. Levin, Nonoccilation of solutions of equation $x^{(n)}+p_{1}(t) x^{(n-1)}+$ $\ldots+p_{n}(t) x=0$, Uspehi Math. Sci., XXIV, 2 (146) (1969), 43-94 (Russian).

[9] G. Mammana, Decomposizione delle espressioni differenziali lineari omogenee in prodotto di fattori simbolici e applicazione relativa allo studio delle equazioni differenzi ali lineari, Math. Z., 33 (1931), 186231 (Italian).

[10] M. Nassyrova, Weighted inequalities involving Hardy-type and limiting geometric mean operators, PhD thesis 2002:3, ISSN:1402-1544, Department of Mathematics, Luleå University of Technology, 2002.

[11] R. Oinarov, Two-sided estimates for the norm of some classes of integral operators, Trudy Mat. Inst. Steklov, 204 (1993), 240-250 (Russian); English transl.: Proc. Steklov Inst. Math., 204 (1994), 205214. 
[12] R. Oinarov and B. O. Sagindykov, Estimate of multiple integration operator with weights on cone of monotone functions in the case $1<q<p<\infty$, Republican Scientific Journal: Science and Education of the South Kazakhstan, Ser.:Mat., Inf., Shymkent, 7(14) (1998), 5360 (Russian).

[13] B. Opic and A. Kufner, Hardy-Type Inequalities, Pitman Research Notes in Mathematics 219, Longman Scientific and Technical, Harlow, 1990.

[14] G. Polya, On the mean-value theorem corresponding to a given linear homogeneous differential equation, Trans. Amer. Math. Soc., 24 (1924), $312-324$.

[15] V. D. Stepanov, Two-weighted estimates for Riemann-Liouville integrals, Report No. 39, Math. Inst., Czechoslovak Acad. Sci., 1988.

Eurasian National University

Munaytpasov St., 5

010000 Astana, Kazakhstan

(E-mail : aigerim_k@academset.kz)

Luleå University of Technology

Department of Mathematics

SE - 97187 Luleå

Sweden

(E-mail : larserik@sm.luth.se) 


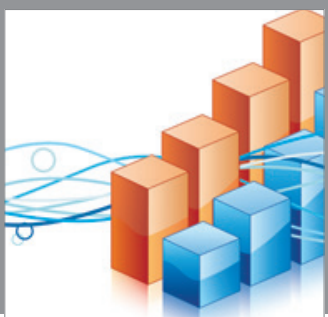

Advances in

Operations Research

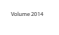

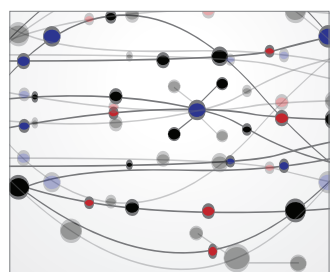

\section{The Scientific} World Journal
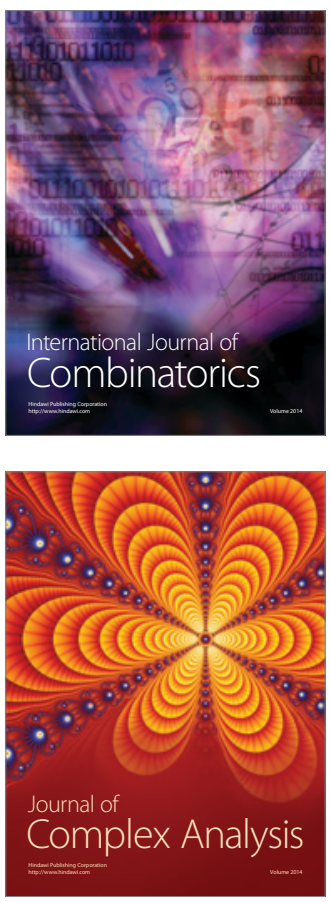

International Journal of

Mathematics and

Mathematical

Sciences
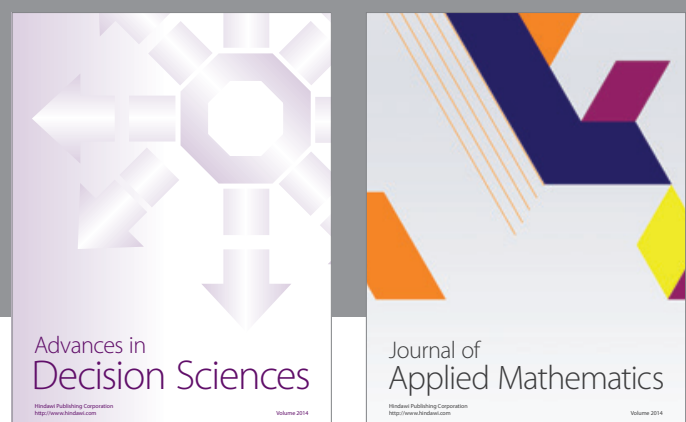

Journal of

Applied Mathematics
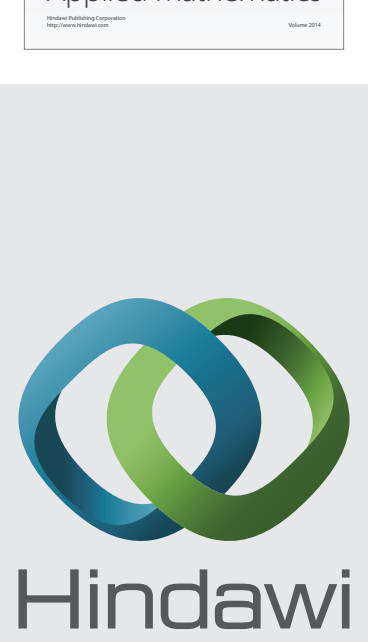

Submit your manuscripts at http://www.hindawi.com
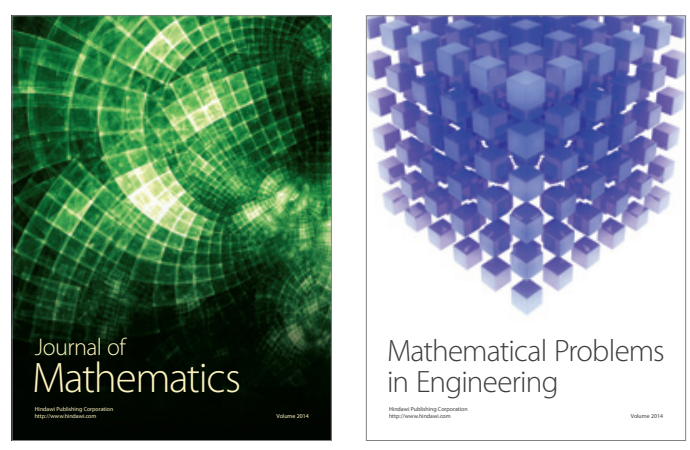

Mathematical Problems in Engineering
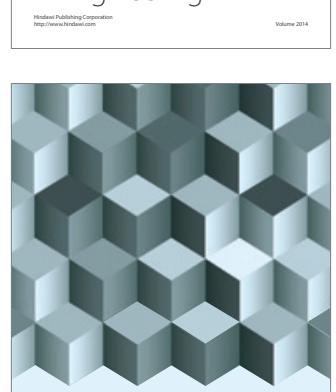

Journal of

Function Spaces
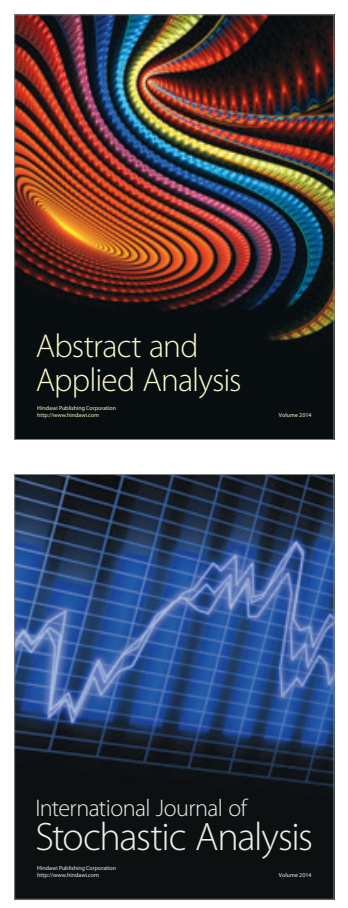

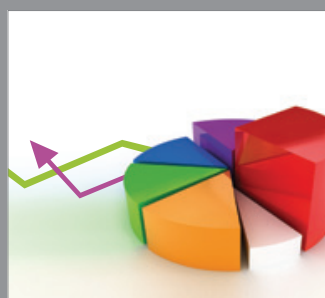

ournal of

Probability and Statistics

Promensencen
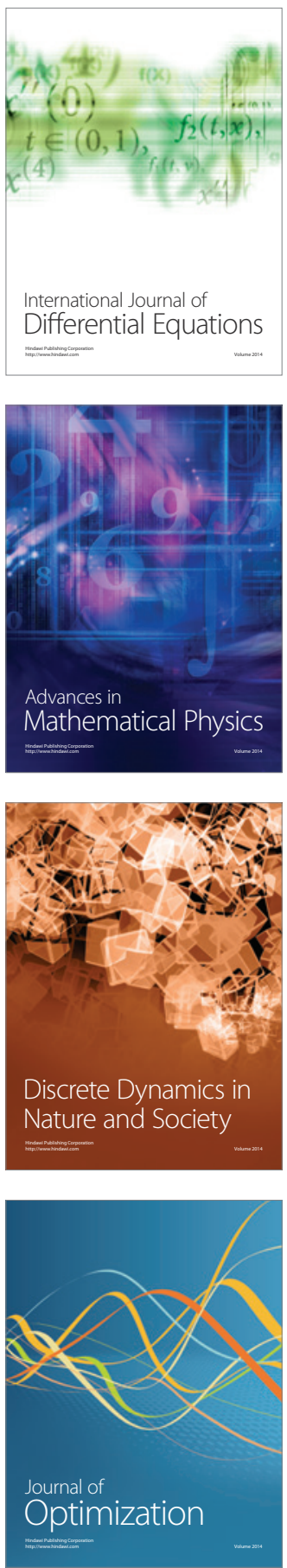